\title{
Statistical Accuracy and CPU Time Characteristic of Three Trajectory Split Methods for Monte Carlo Simulation of Ion Implantation
}

\author{
W. Bohmayr ${ }^{a}$, A. Burenkov ${ }^{b}, J$. Lorenz $^{b}$, H. Ryssel ${ }^{b, *}$, and S. Selberherr ${ }^{a}$ \\ ${ }^{a}$ Institute for Microelectronics, TU Vienna \\ Gusshausstrasse 27-29, A-1040 Vienna, Austria \\ ${ }^{b}$ Fraunhofer-Institut für Integrierte Schaltungen. \\ Schottkystrasse 10, 91058 Erlangen, Germany \\ *Lehrstuhl für elektronische Bauelemente, Universität Erlangen-Nürnberg, \\ Cauerstrasse 6, 91058 Erlangen, Germany
}

\begin{abstract}
Three trajectory split methods [1] for the acceleration of two and three-dimensional Monte Carlo simulation of ion implantation into crystalline targets are presented. They ensure a much better statistical representation in regions with a dopant concentration several orders of magnitudes smaller than the maximum. As a result the time required to perform a simulation with comparable statistical accuracy is drastically reduced. The advantages of the new approaches have been confirmed by a thorough statistical analysis.
\end{abstract}

\section{Introduction}

Inspired by the results in [2] about the rare event approach implemented in the UT-MARLOWE code [3] for one-dimensional structures. we developed the trajectory split method [1] for the Monte Carlo simulation of ion implantation which drastically reduces the computational effort and is applicable for two and three-dimensional simulations. A similar method was first used in the work of Phillips and Price [4] to simulate hot electron transport.

\section{The Trajectory Split Method}

The traditional Monte Carlo approach for crystalline targets is based on the calculation of a large number of "distinct" ion trajectories, i.e. each trajectory is usually followed from the ion starting point at the surface of the target up to the stopping point of the ion. Since the majority of ion trajectories ends at the most probable penetration depth, the statistical noise of regions with a dopant concentration several orders of magnitudes smaller than the maximum (in the following we call these 
areas "peripheral") cannot be tolerated and we have to increase the total number of calculated ions.

The fundamental ideas of our new simulation approach are to locally increase the number of calculated ion trajectories in areas with large statistical uncertainty and to utilize the information we can derive from the flight-path of the ion up to a certain depth inside the target. For each ion, the local dopant concentration $\mathrm{C}_{\text {loc }}$ is checked at certain points of the flight-path (checkpoints). At each checkpoint we relate $\mathrm{C}_{\text {loc }}$ to the current maximum global concentration $\mathrm{C}_{\max \text {,current }}$ by calculating the ratio $\mathrm{C}_{\text {loc }} / \mathrm{C}_{\max \text {,current. }}$ The result is compared with given relative concentration levels (we define ten levels at $\left.0.3,0.09,0.027, \ldots, 0.3^{10}\right)$. Only if the current local concentration falls in an interval below the previous one, a trajectory split point is defined at this checkpoint. Therefore our approach is a self-adaptive algorithm because more split points are defined at areas with unsatisfying statistical accuracy. Additional trajectory branches are suppressed, if an ion moves from lower to higher local concentration levels. We store the position of the ion, its energy as well as the vector of velocity and use this data for virtual branches of ion trajectories starting at this split point. In this way, the peripheral areas of the dopant concentration are represented by a much higher number of ion trajectories and the statistical noise is reduced.

\section{Three Split Strategies and their Statistical Characteristic}

In our first attempt [1] we chose the simplest implementation of this method by suppressing recursive splits and by permitting only two virtual branches at each split point (Fig. 1). Such a virtual trajectory branch is calculated with the same models and parameters as a regular trajectory, but it starts at the split point with initial conditions obtained from the regular ion. To obtain the correct concentration, a weight is assigned to each branch. The different realizations of the virtual trajectories result from the thermal vibrations of the target atoms [5].

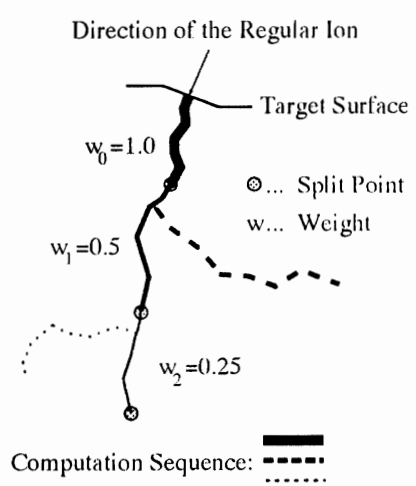

Figure 1: Topological structure of the non-recursive split method, the weight of each branch, and the sequence of its calculation

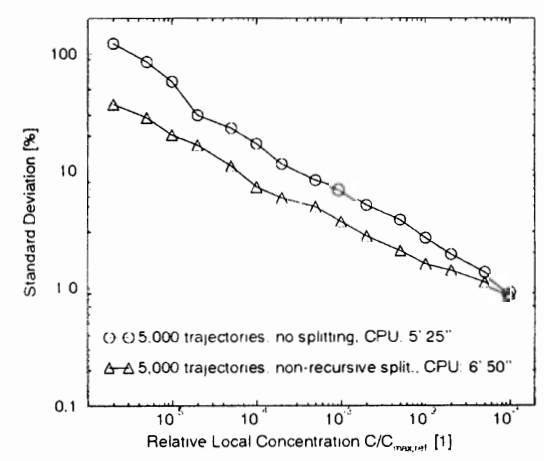

Figure 2: Two-dimensional point response of phosphorus implant, statistical accuracy and CPU time of nonrecursive split method

In the present work we present two additional trajectory split methods: recursive Trajectory $\underline{R}$ elated $\underline{S}$ plit method (TRS, Fig. 3) and recursive $\underline{S}$ plit-level $\underline{R}$ elated $\underline{\text { Split }}$ 
method (SRS, Fig. 5). As an important example, we perform a Monte Carlo simulation of a phosphorus implant at $50 \mathrm{keV}$ into (100) oriented single-crystal silicon covered by $2.5 \mathrm{~nm}$ of oxide to obtain point response distributions. The required computational effort for such a simulation is about 70 minutes for the conventional approach and about 12 minutes for the new trajectory split methods using a HP 735/100 workstation. Implantation damage formation has been neglected.

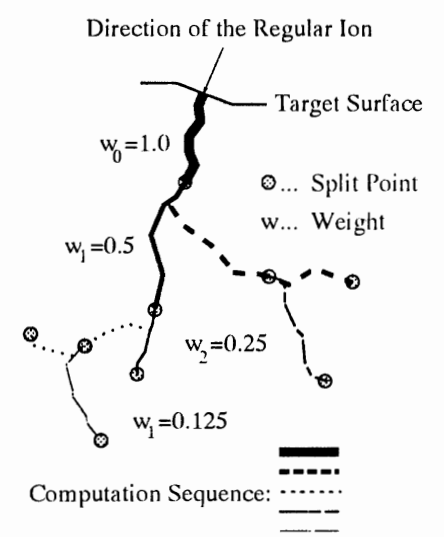

Figure 3: Topological structure of the trajectory related method (TRS), the weight of each branch, and the sequence of its calculation

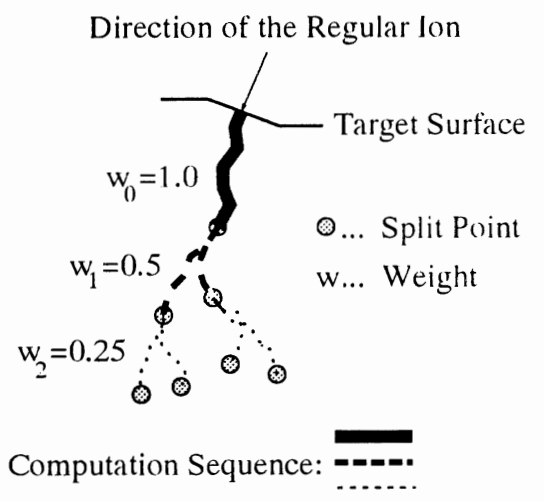

Figure 5: Topological structure of the split-level related split method (SRS). the weight of each branch, and the sequence of its calculation

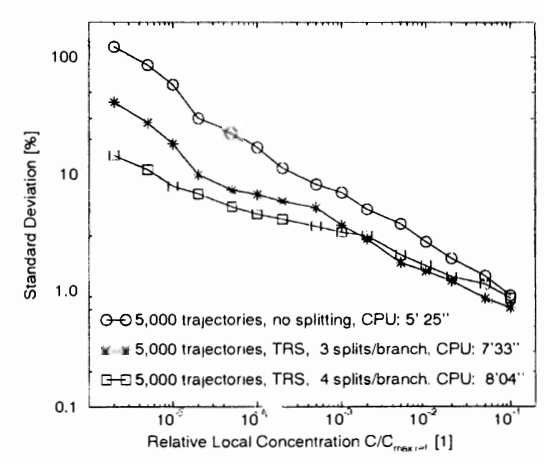

Figure 4: Two-dimensional point response of a phosphorus implant, statistical accuracy and CPU time of trajectory related split method (TRS)

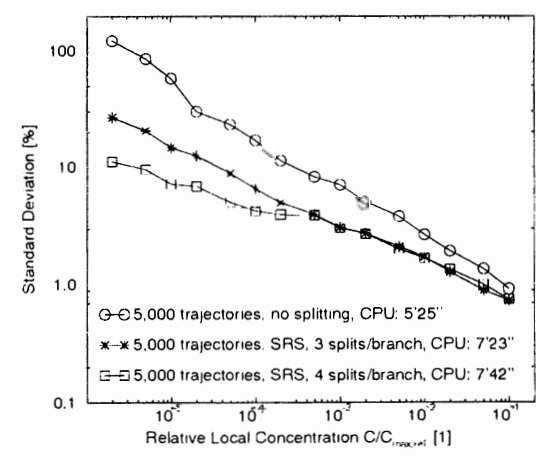

Figure 6: Two-dimensional point response of a phosphorus implant, statistical accuracy and CPU time of split-level related split method (SRS)

To assess the statistical accuracy of the results obtained from the conventional and from the trajectory split methods, we define a mean-square deviation from a reference distribution. For that reason we carry out a conventional simulation result with such a high number of ion trajectories $(1,000,000)$ that statistical fluctuations are negligible in the concentration area considered. 


\section{Simulation Results}

We present the deviation data for the non-recursive (Fig. 2), TRS (Fig. 4), and SRS method (Fig. 6) calculated with 5,000 distinct ion trajectories. The relative concentration in these figures is defined as the ratio of $\mathrm{C} / \mathrm{C}_{\max , \text { ref }}$, where $\mathrm{C}_{\max , \text { ref }}$ means the maximum concentration of the reference distribution. It is evident that the relative standard deviation decreases for high local concentrations. Therefore areas with low local concentration are well suited to demonstrate the merits of the new simulation strategy. The differences in Fig. 4 and Fig. 6 result from slightly modified split algorithms. Our approaches show a deviation from the reference distribution which is up to ten times smaller compared to the deviation of the standard method. The computational effort is approximately proportional to the number of distinct ion trajectories and the additional overhead due to trajectory splits is only $25 \%$ to $35 \%$. Further important advantages of the trajectory split method are its lower sensitivity to the local concentration and the opportunity to individualize its error behavior. Increasing the number of splits per branch (Fig. 4 and Fig. 6) and/or initalizing more than one virtual branch at each split point leads to a significantly smaller error in peripheral areas without effecting the statistic in other regions. In other words, there is a chance of optimizing the relation between $\mathrm{CPU}$ time and required statistical accuracy for a particular problem.

It should be mentioned that our new strategy is also well suited to compute the collision cascade of a displaced target atom ("recoil") because it offers the possibility to optimize the recoil statistic by a random deletion of recoil trajectories at places with a statistical "over-representation" and by splitting them at peripheral areas of the collision cascade. It is also expected that the $S R S$ method will prove to be more profitable if implantation damage is considered.

\section{Acknowledgement}

Part of this work was carried out in cooperation between PROMPT (JESSI project BT8B) and ADEQUAT (JESSI project BT11) and has been funded by the EU as ESPRIT projects No. 8150 and 8002 , respectively. TU Vienna wants to acknowledge important support by Digital Equipment Corporation, Hudson, USA.

\section{References}

[1] W. Bohmayr and S. Selberherr, "Trajectory Split Method for Monte Carlo Simulation of Ion Implantation Demonstrated by Three-Dimensional Poly-Buffered LOCOS Field Oxide Corner", VLSI-TSA, Taipei, Taiwan, 1995.

[2] S.-H. Yang, D. Lim, S. Morris, and A.F. Tasch, "A More Efficient Approach for Monte Carlo Simulation of Deeply-Channeled Implanted Profiles in Single-Crystal Silicon", NUPAD, pp. 97-100, 1994.

[3] K.M. Klein, C. Park, and A.F. Tasch, "Monte Carlo Simulation of Boron Implantation into Single-Crystal Silicon", IEEE Trans. Electron Devices, ED-39, pp. 1614-1621, 1992.

[4] A. Phillips and P.J. Price. "Monte Carlo Calculations on Hot Electron Energy Tails", Applied Physics Letters, vol. 30, no. 10, pp. 528-530. 1977.

[5] M. Jaraiz, J. Arias, E. Rubio, L.A. Marques, L. Pelaz, L. Bailon, and J. Barbolla, "Dechanneling by Thermal Vibrations in Silicon Ion Implantation", X International Conference on Ion Implantation Technology, Abstract P-2.19, Catania, Italy, 1994. 